\title{
The Conformational Transition in the Concentrated Solution of Sodium Poly(L-glutamate)
}

\author{
Yoshiharu Tsujita, Ikuo YAmanAKA, and Akira TAKIZAwA \\ Nagoya Institute of Technology, Department of Fiber and Polymer Engineering, \\ Gokiso-cho, Showa-ku, Nagoya 466, Japan.
}

(Received January 31, 1979)

\begin{abstract}
The conformational transition (random coil $-\alpha$ helix $-\beta$ form) of a sodium poly(Lglutamate) has been examined in solution at various concentrations and temperatures by infrared absorption spectra, X-ray diffraction, differential scanning calorimetry, and polarized microscopy. At room temperature, random coil conformation is retained at a dilute concentration below $40 \%$, and the $\alpha$ helix is formed within a concentration range from $45-70 \%$, and at a concentration above $75 \%$, the $\beta$ form is a stable conformation. Either the thermal transition of the $\alpha$ helix-random coil or the $\alpha$ helix $-\beta$ form is accompanied with the endothermic peak in a thermogram depending on the solution concentration. Also, within a concentration range from $45-70 \%$, the concentrated solution forms the anisotropic phase which consists the $\alpha$ helix, i.e., the lyotropic cholesteric liquid crystal. The anisotropic phase transforms into the isotropic phase with increase in temperature. Spherulites consisting of the $\beta$ form conformation and which include water between the sheets of the $\beta$ form could be observed at a concentration above $75 \%$.

KEY WORDS $\quad \alpha$ Helix / $\beta$ Form / Random Coil / Conformational Transition

/ Transition Enthalpy / Anisotropic Solution / Liquid Crystal /
\end{abstract}

Many studies on a concentrated solution of polypeptide-organic solvent system have shown that the concentrated polypeptide solution forms the lyotropic-type liquid crystal of the cholesteric and nematic liquid crystal. ${ }^{1-4}$ Although the origin of a twisting in the cholesteric liquid crystal is not clear, the temperature dependence and the concentration dependence of the pitch characteristics of twisted structures have been established experimentally. However, there are but few studies on concentrated solutions of the polypeptide-water system and these are of interest in the light of biopolymers. Sodium poly(L-glutamate) (SPG) is one of the water-soluble polypeptides and may be considered as a model protein compound in a concentrated aqueous solution.

In a dilute aqueous solution, SPG retains its random-coil conformation above $\mathrm{pH} 7$ because of the intramolecular electrostatic repulsion between anions at the end of the side chain. ${ }^{5}$ Below $\mathrm{pH} 7$, ionized SPG takes on to a leser extent the $\alpha$ helix conformation in a dilute aqueous solution. On the other hand, Keith et al ${ }^{6.7}$ have studied the structure of the solid state of poly(L-glutamic acid) and its alkaline earth salts, and have reported that such polypeptides form the crystal structure of the $\beta$ form in the solid state. Thus it is expected that the conformational transition from either the ionized random-coil conformation or the lesser ionized $\alpha$ helix conformation in a dilute aqueous solution to the $\beta$ form in the solid state may occur with increase in concentration. It is interesting to examine the conformational change induced by variations in the concentration and also the temperature in concentrated aqueous solutions.

In this paper, the conformational transition of SPG is studied at various high concentrations and at various temperatures. The anisotropic phase in the concentrated aqueous solution is also examined by $\mathrm{X}$-ray diffraction and polarized microscope.

\section{EXPERIMENTAL}

SPG having a molecular weight of approximately 25,000 was kindly supplied by Kyowa Hakko Co. Inc. Homogeneous solution of a concentration ranging from $45-55 \%$ was prepared by an equilibrium sorption of water vapor onto a dry solid film which 
had been cast from a 3\% SPG-water solution. Specimen at a concentration above $55 \%$ was prepared by equilibrium desorption of the homogeneous solution obtained above. Also, this concentrated solution was stored for a few days with variation in concentration and the homogeneous concentrated solution can be obtained. ${ }^{8}$ The concentration $(\mathrm{w} / \mathrm{w})$ of polymer was determined by weighting the amount of sorbed water and a dry solid film.

Infrared absorption spectra were measured in the wave number range from $4,000 \mathrm{~cm}^{-1}$ to $400 \mathrm{~cm}^{-1}$ using a diffraction-grating infrared spectrophotometer (IRA-2, manufactured by Japan Spectroscopic Co. Ltd.). Utilizing an attenuated total reflection (ATR) prism, spectra of the concentrated solution were obtained and are shown without correction for absorption of water and the ATR prism.

$\mathrm{X}$-ray diffraction photographs of the concentrated solution were taken with a flat plate camera at room temperature. During an X-ray radiation time of several hours, change in the concentration due to evaporation of water must be avoided. Thus, the concentrated aqueous solution was wrapped with a very thin polyester film to keep the concentration almost constant. Spacings were evaluated from Debye rings observed on the X-ray diffraction photograph. The camera distance from the specimen to the film was calibrated by silicon powder.

The specimen $(10-20 \mathrm{mg})$ used for differential scanning calorimeter (DSC) measurement was put into an aluminum pan and sealed up tightly to prevent any change in concentration during scanning. The DSC used in this study was the DSC standard type of the Rigaku Denki Co. Ltd. The sensitivity of the DSC measurement was $\pm 2 \mathrm{mcal} \mathrm{s}^{-1}$ $\left(8.4 \mathrm{~m} \mathrm{Js}^{-1}\right)$ and the scanning rate was $5^{\circ} \mathrm{C} \mathrm{min}^{-1}$. The experimental error of the transition enthalpy was due mostly to ambiguous determination of the base line in the DSC thermograms and was about $\pm 5 \%$.

\section{RESULTS AND DISCUSSION}

Infrared absorption spectra of $60 \%$ SPG solution and $100 \%$ SPG solution, i.e., solid SPG are shown in Figure 1. Both spectra are distinctive and the characteristic Amide-I band of $60 \%$ and $100 \%$ SPG solution is located at the wave numbers $1640 \mathrm{~cm}^{-1}$ and

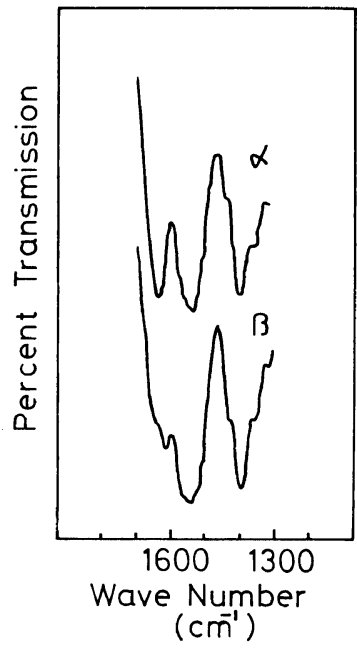

Figure 1. Infrared absorption spectra of $60 \%$ SPG solution $(\alpha)$ and $100 \%$ SPG solution (solid state) $(\beta)$.

$1620 \mathrm{~cm}^{-1}$, respectively, suggesting a conformational difference between two solutions. Lenormant et al. ${ }^{9}$ have studied the conformational change in SPG at various relative humidities and reported that in a relative humidity range from $30-70 \%$, the $\beta$ form is retained and above $90 \%$, the $\alpha$ form is stable. Our results agree with their results in the infrared absorption spectra, especially, in the wave number of the Amide-I band. Infrared absorption spectra of $60 \%$ SPG solution in Figure $1(\alpha)$ exhibit the $\alpha$-helix conformation which is the same as the $\alpha$ form denoted by Lenormant et al., ${ }^{9}$ and the spectra of $100 \%$ SPG solution in Figure $1(\beta)$ show the $\beta$ form. It was found that the $\beta$ form was stable in a very concentrated solution and in the solid state, and that the $\alpha$ helix was a stable conformation in the vicinity of the concentration $60 \%$. But in a dilute solution, the random-coil conformation is well known to be formed above $\mathrm{pH} \mathrm{7.5}$ The conformational transition (random coil- $\alpha$ helix $-\beta$ form) is, therefore, expected as concentration increases.

The structure of the SPG solution at a concentration above $55 \%$ was studied by X-ray diffraction analysis. Spacings evaluated from the diffraction rings obtained are shown in Table I and Figure 2. The relationship between the spacings and the concentration does not change continuously in the vicinity of a concentration $75 \%$. This indicates a structural difference in the SPG solution of the concentration above and that below $75 \%$. By com- 
The Conformation of Sodium Poly(L-glutamate)

Table I. Spacings obtained from X-ray diffraction analysis in a concentration range from $55 \%$ to $100 \%$

\begin{tabular}{|c|c|c|c|c|c|c|}
\hline Concn $/ \%$ & & & & $s / \AA$ & & \\
\hline 55.1 & $\begin{array}{c}15.10 \\
\text { (s) }\end{array}$ & $\begin{array}{l}8.78 \\
\text { (m) }\end{array}$ & $\begin{array}{l}7.54 \\
\text { (w) }\end{array}$ & & & \\
\hline 62.0 & $\begin{array}{c}14.44 \\
(\mathrm{~m})\end{array}$ & $\begin{array}{l}8.29 \\
\text { (m) }\end{array}$ & $\begin{array}{l}7.20 \\
\text { (w) }\end{array}$ & & & \\
\hline 70.6 & $\begin{array}{c}14.00 \\
\text { (w) }\end{array}$ & $\begin{array}{l}8.05 \\
\text { (w) }\end{array}$ & $\begin{array}{l}7.13 \\
(\mathrm{vw})\end{array}$ & & & \\
\hline 81.1 & & & & $\begin{array}{l}7.31 \\
\text { (w) }\end{array}$ & $\begin{array}{c}4.80 \\
(\mathrm{~s})\end{array}$ & \\
\hline 92.2 & $\begin{array}{c}13.54 \\
(\mathrm{vw})\end{array}$ & & & $\begin{array}{l}6.65 \\
\text { (m) }\end{array}$ & $\begin{array}{c}4.78 \\
(\mathrm{~s})\end{array}$ & $\begin{array}{l}4.41 \\
(\mathrm{vw})\end{array}$ \\
\hline 100.0 & $\begin{array}{c}13.48 \\
(\mathrm{vw})\end{array}$ & & & $\begin{array}{l}6.57 \\
(\mathrm{~m})\end{array}$ & $\begin{array}{l}4.71 \\
(\mathrm{vw})\end{array}$ & $\frac{-}{(\mathrm{vvw})}$ \\
\hline
\end{tabular}

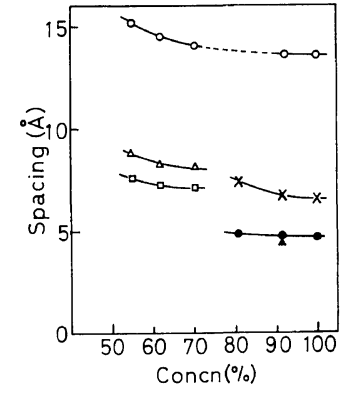

Figure 2. Plots of the spacings and the concentration of SPG solution.

parison of the spacings of the $\alpha$ and the $\beta$ forms in the solid state reported by Mitsui ${ }^{10}$ with spacings obtained here in the concentrated solution, at a concentration below $70 \%$, the $\alpha$ helix is considered to be hexagonally packed to form the $\alpha$ form and above $75 \%$, the $\beta$ form comes about. The spacings of the SPG solution at concentrations of $92.2 \%$ and $100 \%$ are 13.54 and $13.48 \AA$, respectively, and correspond to the $\alpha$ form which remained in part during preparation of the film. The $\alpha$ form is not essentially stable at these concentrations, although Mitsui prepared the $\alpha$ form in the solid state. Therefore, the spacings of the $\alpha$ form should not be observed in this concentration range. The spacings, except for the $4.7 \AA$ spacing, gradually decrease with increase in concentration as shown in Figure 2. This behavior for both forms is thought to be caused by dilution by water. The dilution for the $\alpha$ form occurs among the $\alpha$ helixes, especially, in the side chain regions. The [100] spacing of the $\alpha$ form increases with dilution, but, for the $\beta$ form, a different type of dilution is characteristic. That is, the $4.7 \AA$ spacing of the solid state, attributed to the spacing between main chains in a direction of hydrogen bonds in the sheet of the $\beta$ form, does not increase significantly as the concentration decreases, and the $6.6 \AA$ inert-sheet spacing of the solid state tends to become larger with an decrease in concentration. Thus, water molecule may not be able to enter or get between the main chains in a sheet of the $\beta$ form, but capable of getting between two sheets.

DSC thermograms of solution of concentration ranging from $45-70 \%$ were measured, and a typical thermogram is drawn in Figure 3. Only the thermog-

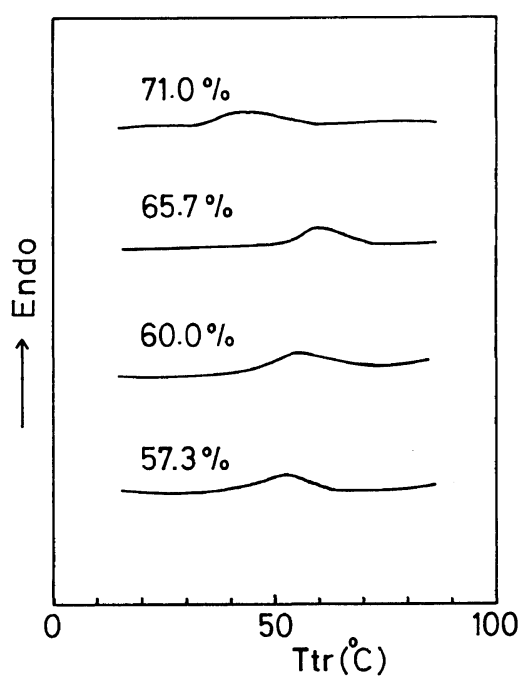

Figure 3. DSC thermograms of SPG solution (a percentage represent the concentration of SPG solution). 


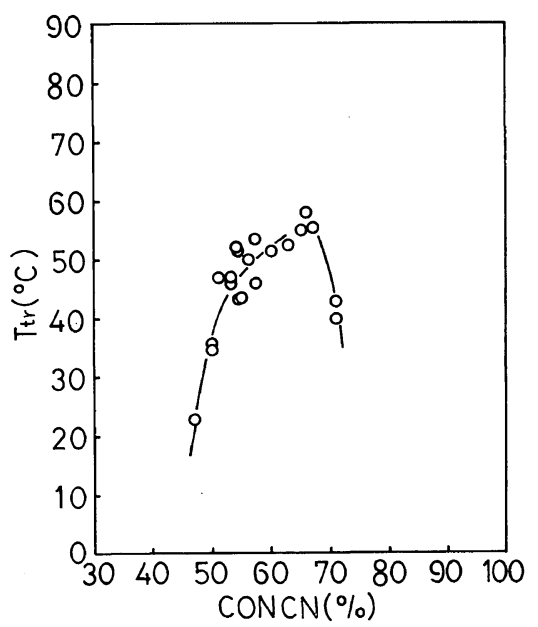

Figure 4. The transition temperature $v s$. the concentration of SPG solution.

ram of the heating curve is represented in this figure, although the thermogram exhibits a curve with a reversible hysteresis loop. An endothermic peak appeared within temperature range from $25-60^{\circ} \mathrm{C}$ and was dependent on concentration. The transition temperature, i.e., the temperature corresponding to maximum of an endothermic peak $v s$. concentration of SPG solution is shown in Figure 4. The transition temperature increases as the concentration is increased from $45-60 \%$ and up to a maximum of $65 \%$, but decreases considerably above this concentration. According to studies on the Amide- $\mathrm{V}$ band of infrared absorption spectra reported by Iizuka et $a l .{ }^{11}$ an isotropic solution is formed above the transition temperature and the conformation in such a solution is confirmed as a random coil at a concentration range of $40-60 \%(\mathrm{w} / \mathrm{v})$. Thus, the endothermic peak appearing in the thermogram of a solution having a concentration ranging from 45 $65 \%$ can be attributed to the $\alpha$-helix-random coil transition. From the fact that the $\beta$ form is a stable form of SPG above the concentration of $75 \%$, the endothermic peak appearing above a concentration of $65 \%$ is thought to be due to the $\alpha-\beta$ transition. The former is the normal (helix to coil) thermal transition different from the inverse transition usually observed in polypeptide-active solvent system. This suggests the helix coil transition of the peptide group (SPG) in the presence of only the inert solvent. The transition enthalpy of the concentrated solution with sufficient aging (about one week) is given in Table II. If aging is insufficient for a solution of 50.1 and $53.0 \%$ concontration the transition enthalpy of such a specimen is two thirds smaller than the value given in Table II. It was found that sufficient aging is necessary to form the $\alpha$ helix sufficiently. The transition temperature, however, was independent of aging.

Table II. The transition enthalpy of SPG solution aged for several days

\begin{tabular}{cc}
\hline Concn $/ \%$ & $H / \mathrm{cal} \mathrm{mol}^{-1}{\text { (of residue })^{\mathrm{a}}}^{\circ}$ \\
\hline 50.1 & 239 \\
53.0 & 350 \\
66.2 & 319 \\
70.8 & 168 \\
\hline
\end{tabular}

a $1 \mathrm{cal} \mathrm{mol}^{-1}=4.184 \mathrm{~J} \mathrm{~mol}^{-1}$

Within a concentration range from $45-65 \%$ at room temperature, the X-ray diffraction rings observed exhibited the hexagonal packing of the $\alpha$ helix described above and this confirms the formation of the anisotropic solution, i.e., liquid crystal. Polarized microscopic observation also showed the existence of the anisotropic phase at this concentration range at room temperature as will be mentioned later. In this concentration range, the transition of the $\alpha$ helix-random coil is accompanied with the transition of the anisotropic solution (liquid crystal) to an isotropic solution. However, the transition enthalpy of the latter has been reported as very small. Johnson et al. ${ }^{12}$ have summarized the calorimetric data for the liquid-crystalline transition and have concluded that the transition enthalpy of the liquid crystal to the isotropic liquid is only 2 or $3 \%$ of the total enthalpy change of the crystal to the isotropic liquid. Probably, a qualitative explanation can be made similarly for the transition of the liquid crystal to the isotropic solution studied here. Thus, the transition enthalpy obtained is attributed primarily to only the transition enthalpy of the $\alpha$ helix-random coil.

Karasz and Gajnos ${ }^{12}$ have studied the thermodynamics of the helix-coil transition taking into consideration the contribution of polypeptides and active solvents forward the helix-coil transition. The helix-coil transition enthalpy of polypeptide itself in the presence of only an inert solvent, $H_{1}\left(H_{\text {coil }}-H_{\text {helix }}\right)$ is, for example, $845 \mathrm{cal} \mathrm{mol}^{-1}\left(3.5 \mathrm{~kJ} \mathrm{~mol}^{-1}\right)$ of re- 
sidue and $390 \mathrm{cal} \mathrm{mol}^{-1}\left(1.6 \mathrm{~kJ} \mathrm{~mol}^{-1}\right)$ of residue for poly $(\gamma$-benzyl glutamate $)$ and poly $(\beta$-benzyl aspartate), respectively. The small value of the transition enthalpy $H_{1}$ of poly $(\beta$-benzyl aspartate) corresponds to the unstability of its $\alpha$ helix which is well known to be more unstable than the $\alpha$ helix of poly ( $\gamma$-benzyl glutamate). Since the SPG-water system studied here is considered to be a polypeptide-inert solvent system, the helix-coil transition enthalpy $H_{1}$ reported by Karasz and Gajnos can be compared to the transition enthalpy obtained in this study. The transition enthalpy obtained in the present study is approximately $250-350 \mathrm{cal} \mathrm{mol}^{-1} \quad(1.0-1.5 \mathrm{~kJ}$ $\mathrm{mol}^{-1}$ ) of residue as shown in Table II. Such a value is rather near the value for poly ( $\beta$-benzyl aspartate), $390 \mathrm{cal} \mathrm{mol}^{-1}\left(1.6 \mathrm{~kJ} \mathrm{~mol}^{-1}\right)$ of residue. Although the structure of SPG is comparable to that of $\operatorname{poly}(\gamma$ benzyl glutamate), the $\alpha$ helix of SPG may be considered as having a stability similar to that of poly $(\beta$-benzyl aspartate) from the stand point of the transition enthalpy $H_{1}$. The reason for the instability of the SPG $\alpha$ helix may be the repulsion and/or the steric hindrance between the carboxylate anions at a low degree of ionization.

The transition of the random coil- $\beta$ form should be expected to occur at a higher temperature range. This transition, however, could not be detected below $95^{\circ} \mathrm{C}$, where DSC measurements were performed in this experiment. Further studies on this transition will be carried out in the future.

Polarized microscopic observations were made at three concentrations as shown in Figure 5. A microphotograph of a solution at a concentration of $41 \%$ exhibits on the whole a dark field for the isotropic phase, including very small parts of the anisotropic phase with little refined texture [Figure $5(\mathrm{a})]$. Solution of a concentration of $41 \%$ is considered to be essentially the isotropic phase. At a greater concentration $(55 \%)$, the amount of the anisotropic phase increases and also the spherulites are incorporated into a larger anisotropic phase [Figure 5(b)]. In this concentration range the conformation of SPG is the $\alpha$ helix as mentioned above. Flory ${ }^{14}$ has proposed from thermodynamic studies the existence of a liquid-crystalline state consisting of rigid rod molecules in the concentrated solution. A concentrated solution of polypeptide-organic solvent system is known to form cholesteric liquid crystals under ordinary conditions and nematic liquid crystals in magnetic or electric fields. The

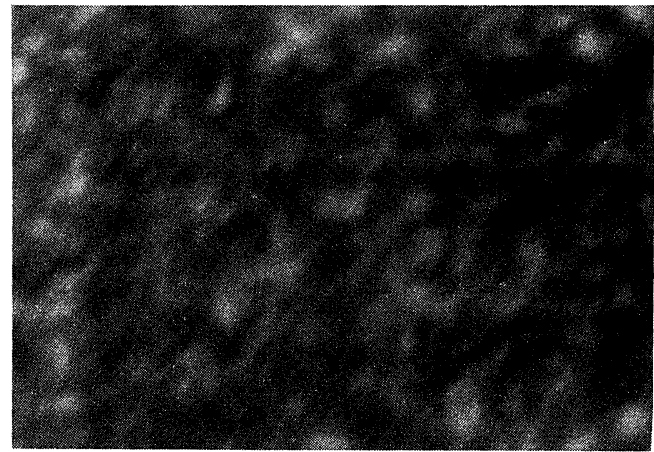

(a)

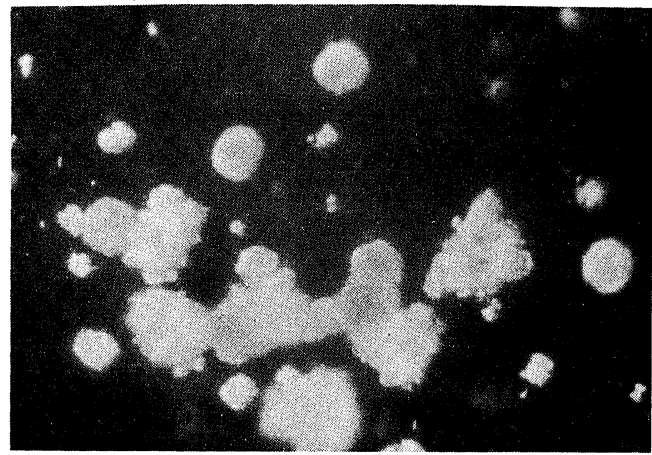

(b)

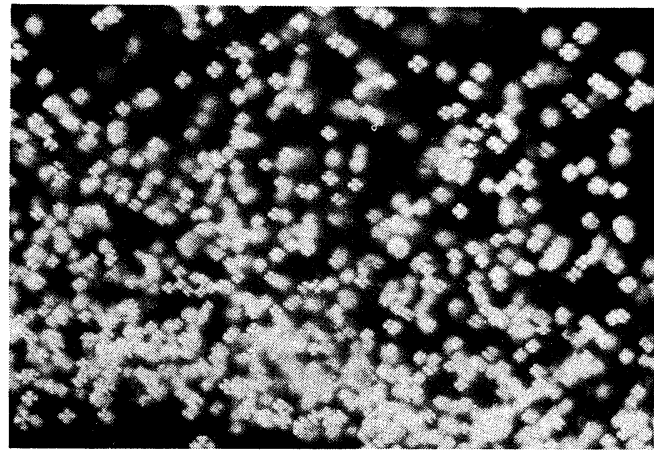

(c)

Figure 5. Polarized microphotographies of SPG solution: (a), concentration $41 \%$; (b), concentration $55 \%$; (c), concentration $72 \%$

anisotropic phase observed in a $55 \%$ concentrated solution may be the cholesteric liquid crystal, judging from small selective reflection appearing in the ultraviolet region in the circular dichroism spectra, ${ }^{15}$ although, by polarized microscope, no retardation line due to the twisted structure of the cholesteric liquid crystal can be observed. However, whether the anisotropic phase is the cholestric liquid crystal or 
not can not be determined only from this result. On the other hand, in a $72 \%$ concentrated solution, SPG retains the $\beta$ form conformation as indicated above and a different microphotograph was taken as shown in Figure 5(c). Spherulites with crossed nicol can be clearly observed and this is a characteristic feature of the $\beta$ form of SPG in the concentrated solution and in the solid state.

\section{CONCLUSION}

In a solution of SPG, ranging in concentration from $45-70 \%$, at room temperature, SPG has the $\alpha$ helix conformation a solution of greater concentration forms the anisotropic phase consisting of the $\alpha$ helix which is hexagonally packed, that is, the lyotropic cholesteric liquid crystal. As the temperature rises, the $\alpha$ helix transforms into either the random coil or the $\beta$-form conformation and also the lyotropic cholesteric liquid-crystal transforms into either the isotropic solution or the $\beta$ form. The spherulites consisting of the $\beta$-form conformation and which include water between the sheets of the $\beta$ form, can be observed at a concentration of $75 \%$.

Acknowledgement. The authors gratefully acknowledge Kyowa Hakko Co. Inc. for suppling sodium poly(L-glutamate). This work was supported by a Grant-in-Aid from the Ministry of Education (no. 335043).

\section{REFERENCES}

1. C. Robinson, Trans. Faraday Soc., 52, 571 (1956).

2. C. Robinson, J. C. Ward, and R. B. Beebers, Discuss. Faraday Soc., 25, 29 (1958).

3. C. Robinson, Tetrahedron, 13, 219 (1961).

4. C. Robinson, Mol. Cryst., 1, 467 (1966).

5. G. D. Fasman, "Poly $(\alpha$-amino acid)s," Marcel Dekker, New York, N.Y., 1967.

6. H. D. Keith, F. J. Padden Jr., and G. Giannoni, J. Mol. Biol., 43, 423 (1969).

7. H. D. Keith, G. Giannoni, and F. J. Padden Jr., Biopolymers, 7, 775 (1969).

8. A. Takizawa, M. Aida, and H. Yamazaki, Bull. Nagoya Inst. Technol., 25, 425 (1973).

9. H. Lenormant, A. Baudras, and E. R. Blout, J. Am. Chem. Soc., 80, 6191 (1958).

10. Y. Mitsui, Biopolymers, 12, 1781 (1973).

11. E. Iizuka, Y. Kondo, and Y. Ukai, Polym. J., 9, 135 (1977).

12. J. F. Johnson, R. S. Porter, and E. M. Barrall II, Mol. Cryst. Liquid Cryst., 8, 1 (1969).

13. F. E. Karasz and G. E. Gajnos, J. Phys. Chem., 77, 1139 (1973).

14. P. J. Flory, Proc. R. Soc. London Ser. A, 234, 73 (1956).

15. H. Saga, Y. Tsujita, and A. Takizawa, unpublished data. 